\title{
Axial Magnetoelectric Effect in Dirac Semimetals
}

\author{
Long Liang $\odot{ }^{1}$ P. O. Sukhachov $\oplus^{2},{ }^{2}$ and A. V. Balatsky ${ }^{1,3}$ \\ ${ }^{1}$ Nordita, KTH Royal Institute of Technology and Stockholm University, Roslagstullsbacken 23, SE-106 91 Stockholm, Sweden \\ ${ }^{2}$ Department of Physics, Yale University, New Haven, Connecticut 06520, USA \\ ${ }^{3}$ Department of Physics and Institute for Materials Science, University of Connecticut, Storrs, Connecticut 06269, USA
}

(Received 13 December 2020; revised 1 March 2021; accepted 18 May 2021; published 17 June 2021)

We propose a mechanism to generate a static magnetization via the "axial magnetoelectric effect" (AMEE). Magnetization $\mathbf{M} \sim \mathbf{E}_{5}(\omega) \times \mathbf{E}_{5}^{*}(\omega)$ appears as a result of the transfer of the angular momentum of the axial electric field $\mathbf{E}_{5}(t)$ into the magnetic moment in Dirac and Weyl semimetals. We point out similarities and differences between the proposed AMEE and a conventional inverse Faraday effect. As an example, we estimated the AMEE generated by circularly polarized acoustic waves and find it to be on the scale of microgauss for gigahertz frequency sound. In contrast to a conventional inverse Faraday effect, magnetization rises linearly at small frequencies and fixed sound intensity as well as demonstrates a nonmonotonic peak behavior for the AMEE. The effect provides a way to investigate unusual axial electromagnetic fields via conventional magnetometry techniques.

DOI: 10.1103/PhysRevLett.126.247202

Introduction.-We are now witnessing a surge of interest in quantum matter with an unusual relativisticlike dispersion relation [1-4]. We mention bosonic (acoustic waves, photonic crystals, and magnons) and fermionic (A phase of superfluid helium-3, $d$-wave superconductors, graphene, topological insulators) Dirac and Weyl materials. The key topological features for all of them are the linear energy spectrum and the presence of several gapless nodes (valleys) that enable exotic artificial fields, which is unique for Dirac materials.

The dynamics of excitations in Dirac and Weyl semimetals and their interplay with electromagnetic fields is a rapidly developing research area. We focus on the inverse Faraday effect (IFE) [5-9] that is a well-known example of light-matter interaction. In this case, the angular momentum from a dynamical electromagnetic (photon) field $\mathbf{E}(t)$ is transferred into the magnetic moment of electrons, leading to a static magnetization $\mathbf{M} \sim \mathbf{E}(\omega) \times \mathbf{E}^{*}(\omega)$. This effect was recently investigated for Dirac and Weyl semimetals in Refs. [10-14].

In this Letter, we propose a mechanism to generate a static magnetization in Dirac and Weyl semimetals through dynamical axial gauge fields, whereby the angular momentum is transferred from the axial or pseudoelectric field $\mathbf{E}_{5}(t)$ into the magnetic moment of electrons

Published by the American Physical Society under the terms of the Creative Commons Attribution 4.0 International license. Further distribution of this work must maintain attribution to the author(s) and the published article's title, journal citation, and DOI. Funded by Bibsam.

$$
\mathbf{M} \sim \mathbf{E}_{5}(\omega) \times \mathbf{E}_{5}^{*}(\omega)
$$

Therefore, we call this phenomenon the "axial magnetoelectric effect" (AMEE). The AMEE is qualitatively different from the IFE. The synthetic nature of the pseudoelectric field in Dirac and Weyl semimetals means that one can induce magnetization using phonons without any electromagnetic fields. This situation reminds us of the difference between the Aharonov-Bohm [15] and Aharonov-Casher [16] effects, where the gauge potentials, while similar, have a different origin.

The origin of the pseudoelectromagnetic fields is rooted in the relativisticlike energy spectrum and topology of Dirac matter. In the case of Dirac semimetals, axial gauge fields could be generated via strains [17-23] as well as magnetization textures $[24,25]$. In graphene, pseudomagnetic fields were observed experimentally in Refs. [26-29]. Previous work focuses mostly on static axial fields. The studies related to the dynamics of these fields have quickly become an active research area. In particular, we mention ultrasonic attenuation [21,30,31], the acoustogalvanic effect [32], and torsion-induced chiral magnetic current [33]. The circularly polarized sound can be generated by acoustic transducers coupled to acoustic quarter-wave plates [34]; see also Refs. [35-37]. In addition to the acoustic method, we also point out recent work on generation of circularly polarized phonons [38]. It has been shown that such phonons can be induced optically [39] in $\mathrm{Cd}_{3} \mathrm{As}_{2}$ [40]. Therefore, the proposed AMEE is timely and instrumental in the elucidation of the axial fields in threedimensional semimetals.

The AMEE is universal in the sense of measuring the response of nodal states to dynamic axial fields regardless 


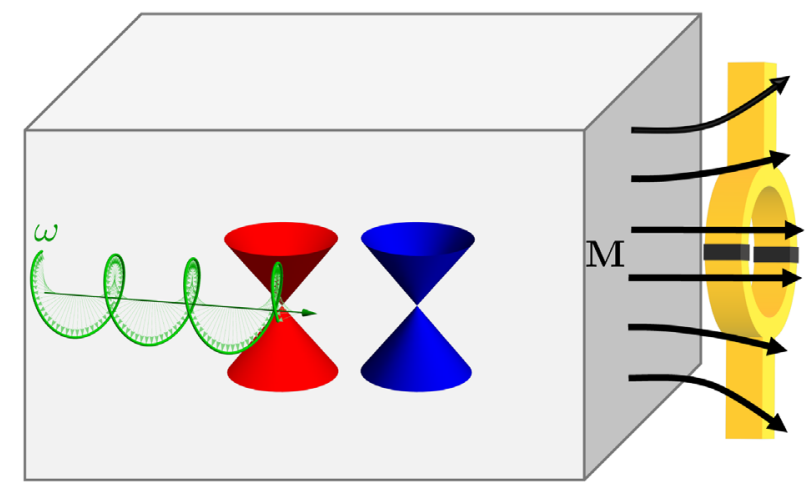

FIG. 1. Schematic model setup for the axial magnetoelectric effect. Circularly polarized axial electric fields lead to the static magnetization $\mathbf{M} \sim \mathbf{E}_{5}(\omega) \times \mathbf{E}_{5}^{*}(\omega)$ of Dirac semimetals. The resulting magnetization is picked up by a magnetometer such as SQUID.

of their origin. Therefore, it could be realized in various experimental setups where the position of the Weyl nodes can be manipulated (for a recent study of the manipulation of the Weyl nodes via light pulses, see Ref. [41]).

By using a dynamical deformation as a characteristic example, we found that the induced magnetization scales as $M \propto \mu \omega$ for small frequencies $\omega$ at fixed sound intensity. This is in drastic contrast to the conventional IFE, where the magnetization scales as $M \propto 1 / \omega$ in the dirty limit. The explanation of this scaling is simple and follows from the nature of the axial electric field. As with conventional IFEs, the induced magnetization scales as the second power of the effective axial electric field $\mathbf{E}_{5}$. Because $\mathbf{E}_{5}$ is proportional to the time derivative of the gauge potentials, which in turn are determined by the strain tensor $u_{i j} \sim \omega$, it results in a two powers of frequency difference when the sound intensity $I \sim \omega^{2}$ is fixed. Hence, we expect that the AMEE has the overall scaling $\sim \omega^{2}$ relative to the IFE for conventional metals. Furthermore, our estimates show that the magnetization induced via the AMEE is within the reach of modern magnetometry technology such as the superconducting quantum interference device (SQUID). The corresponding model setup is schematically shown in Fig. 1.

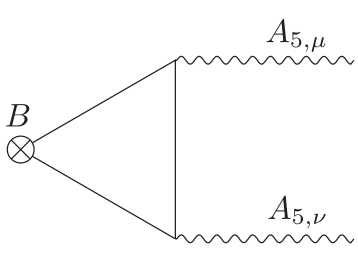

FIG. 2. The Feynman diagram used to calculate the AMEE. The cross denotes an external magnetic field. The wavy lines represent the axial gauge fields.

General theory.-For weak deviations from equilibrium, the magnetization is obtained as a functional derivative of the effective action $S$ with respect to a magnetic field $\mathbf{M}=-\lim _{B \rightarrow 0} \delta S / \delta \mathbf{B}$. (See Fig. 2 for the corresponding Feynman diagram.) Here, $A_{5, \mu}=\left\{A_{5,0},-\mathbf{A}_{5}\right\}$ is the axial gauge field that gives rise to a time-dependent axial electric field $\mathbf{E}_{5}=-\partial_{t} \mathbf{A}_{5}-\boldsymbol{\nabla} A_{5,0}$. While the diagram in Fig. 2 resembles that for the chiral anomaly, it has a different structure, where the external lines correspond to two axial and one vector gauge fields. Therefore, the chiral anomaly action does not contribute to the magnetization. This allows us to calculate the effect separately for each Weyl node. We further assume that the wave vector of the chiral electric field is small enough to ignore the internode transitions.

In general, there could be first-order effects, i.e., $M \sim E_{5}$. They, however, produce an oscillating magnetization that averages to zero for dynamical axial electric fields as well as requires an explicit breakdown of the time-reversal symmetry. Therefore, we focus on a second-order rectification effect, which gives a static magnetization even in a time-reversal symmetric system. This fact makes the AMEE universal and attractive from a practical point of view. For example, while the first-order effects cancel in Dirac semimetals, the AMEE survives.

Without the loss of generality, we assume that $\mathbf{E}_{5}$ is polarized in the $x-y$ plane. Then, the magnetization for a single node is

$$
M_{z}=-i \chi(\omega, \boldsymbol{q})\left(\mathbf{E}_{5, \omega, \boldsymbol{q}} \times \mathbf{E}_{5,-\omega,-q}\right)_{z},
$$

with the response function $\chi(\omega, \boldsymbol{q})$ given by

$$
\begin{aligned}
\chi(\omega, \boldsymbol{q})= & \frac{e^{3}}{4 \omega^{2}}\left\{\sum_{m} \int d \boldsymbol{k} I_{m m m}(\omega, \boldsymbol{q})\left(v_{y}^{m} v_{x}^{m} \partial_{k_{x}} v_{y}^{m}-v_{y}^{m} v_{y}^{m} \partial_{k_{x}} v_{x}^{m}\right)\right. \\
& \left.+\sum_{l \neq m} \int d \boldsymbol{k} I_{m m l}(\omega, \boldsymbol{q})\left(\epsilon_{l}-\epsilon_{m}\right)\left[\left(\epsilon_{l}-\epsilon_{m}\right)^{2}\left(\Omega_{x y}^{m}\right)^{2}+v_{x}^{m} v_{x}^{m} g_{y y}^{m}-v_{x}^{m} v_{y}^{m} g_{x y}^{m}\right]+(x \leftrightarrow y)\right\} .
\end{aligned}
$$

Here, $e$ is the electric charge, the expression for $I_{m n l}(\omega, \boldsymbol{q})$ is given in the Supplemental Material (SM) [42], $m= \pm$ denotes the electron and hole bands, $\epsilon_{ \pm}= \pm \sqrt{v_{i}^{2} k_{i}^{2}}$ is the energy dispersion, $v_{i}^{m}=\partial_{k_{i}} \epsilon_{m}$ is the quasiparticle velocity, and $\Omega_{x y}^{m}$ and $g_{i j}^{m}$ are the Berry curvature [48] and the quantum metric [49] of the band, respectively (see the SM [42] for the explicit expressions). The first term in the curly brackets is the intraband contribution, which 
depends only on the dispersion. The second term originates from interband processes. In this Letter, we focus on the orbital contribution to the magnetization. The spin contribution is estimated to be weak for intraband processes: $\omega \ll 2|\mu|$ (see also Ref. [14] for the IFE results).

The minimal Weyl Hamiltonian

$$
H=\lambda \sum_{i} v_{i} \sigma_{i} p_{i}
$$

was used in the derivation of the response function $\chi(\omega, \mathbf{q})$. Here, $\lambda= \pm$ denotes the chirality of Weyl nodes, $\sigma_{i}$ are the Pauli matrices, $\boldsymbol{p}=-i \boldsymbol{\nabla}$ is the momentum operator, and $\hbar$ and the light velocity $c$ are taken to be unity throughout this Letter. The description of the axial gauge fields $A_{5,0}$ and $\mathbf{A}_{5}$, in general, requires more complicated models where there is a separation between Weyl nodes in momentum space and/or energy. For example, $\mathbf{A}_{5}$ appears in a Weyl semimetal with a broken time-reversal symmetry. We stress, however, that the AMEE is general and does depend on the presence of $\mathbf{E}_{5}$ but not the origin of the axial gauge fields: these gauge fields can be generated by light, strain, or other means. In addition, we notice that all nodes contribute additively to the magnetization (see the SM [42]).

The magnetization given in Eq. (2) with the response function provided in Eq. (3) represents the first main result of this Letter. The response function $\chi(\omega, \boldsymbol{q})$ can be applied in both clean and dirty limits and is also valid for two-dimensional gapless or gapped Dirac semimetals. Furthermore, the result in Eq. (3) is applicable to both conventional inverse Faraday and the axial magnetoelectric effects in Dirac materials.

For a single Weyl node, we find in the $\boldsymbol{q}=0$ limit

$\chi=\frac{e^{3} v_{x} v_{y} \mu}{6 \pi^{2} v_{z} \omega}\left[\frac{1}{\left(\omega+\frac{i}{\tau}\right)^{2}}+\frac{3}{\left(\omega+2 \mu+\frac{i}{\tau}\right)\left(\omega-2 \mu+\frac{i}{\tau}\right)}\right]$,

where $\mu$ is the chemical potential, and $\tau$ is the scattering time. Figure 3 shows the real part of $\chi$ as a function of $\omega / \mu$ for different scattering times. In the $\omega \tau \gg 1$ limit, the first term in Eq. (5), which is the intraband contribution, reduces to the result obtained using the semiclassical theory [12]. In the dirty limit $\omega \tau \ll 1$, the intraband term changes sign. Note that in this limit the semiclassical treatment does not apply. The interband contribution diverges at $\omega=2 \mu$ in the clean limit, and this divergence disappears when the scattering time is finite. In contrast, $\chi$ diverges in the small $\omega$ limit even for finite scattering time. The induced magnetization vanishes when the chemical potential crosses exactly the nodal point. The reason is that the system has particle-hole symmetry at this point. Under particle-hole transformation, the current changes sign, while the axial current remains unchanged [50], and therefore the correlation function $\left\langle j_{5, \mu} j_{5, \nu} j_{\rho}\right\rangle$ that

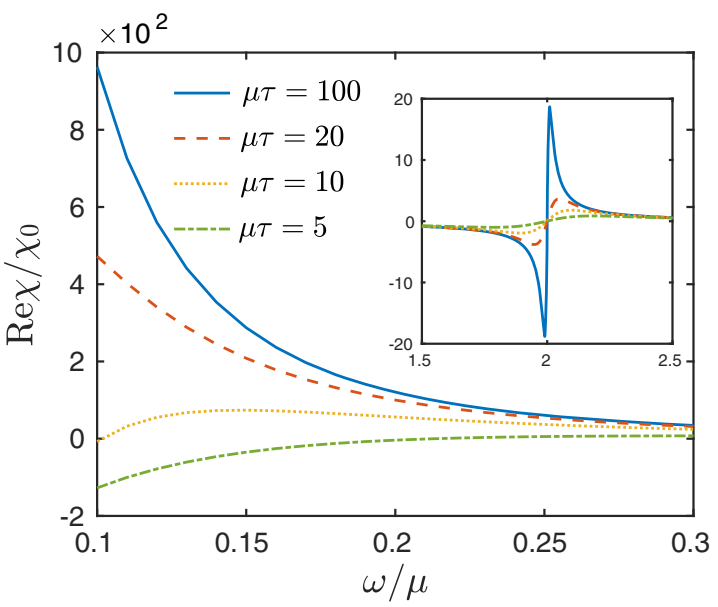

FIG. 3. The frequency dependence of the real part of the normalized response function $\chi / \chi_{0}$ for different scattering times. The inset shows the results for large frequencies $\omega \sim 2 \mu$, where the interband effects are important. Here, $\chi_{0}=e^{3} v_{x} v_{y} /\left(6 \pi^{2} v_{z} \mu^{2}\right), \mu$ is the chemical potential, and $\tau$ is the scattering time.

determines the magnetization (see Fig. 2 and the SM [42]) vanishes identically.

Strain-induced AMEE.-As an example, we calculate the AMEE in strained Dirac semimetals. The axial gauge field is related to the strain fields as [20]

$$
A_{5, i}=\frac{b}{e}\left[\beta u_{i z}+\tilde{\beta}(b) \delta_{i z} \sum_{j} u_{j j}\right],
$$

where the Weyl nodes are separated along the $z$ direction, $\beta$ and $\tilde{\beta}(b)$ are related to the Grüneisen constants, and $u_{i j}=$ $\left(\partial_{i} u_{j}+\partial_{j} u_{i}\right) / 2$ is the strain tensor with $\boldsymbol{u}$ being the displacement field. As an example, we consider a circularly polarized sound wave propagating in the $z$ direction,

$$
\boldsymbol{u}=\operatorname{Re}\left[u_{0}\left(\mathbf{e}_{x}-i \mathbf{e}_{y}\right) e^{i\left(q_{z} z-\omega t\right)}\right],
$$

where $\mathbf{e}_{x}$ and $\mathbf{e}_{y}$ are unit vectors in the $x$ and $y$ directions as well as $\omega=v_{s} q_{z}$ with the transverse sound velocity $v_{s}$.

Then the axial vector potential is

$$
\mathbf{A}_{5}=i\left(\mathbf{e}_{x}-i \mathbf{e}_{y}\right) \frac{b \beta u_{0}}{4 e} q_{z} e^{i\left(q_{z} z-\omega t\right)}+\text { c.c. },
$$

which gives rise to a circularly polarized axial electric field. Note that since $A_{5} \propto q_{z} \propto \omega$, the axial electric field is proportional to $\omega^{2}$.

Since the sound velocity is much smaller than the Fermi velocity $\omega \ll v_{F} q_{z}$, we can no longer take the $q_{z}=0$ limit as for a conventional IFE. Because the sound frequency is small compared to the relaxation timescale, the most experimentally relevant regime is $\omega \tau \ll 1$. On the other hand, since $v_{z} / v_{s} \gg 1$, the condition $q_{z} v_{z} \tau \ll 1$ can be 
easily violated. For example, if we take $\omega=1 \mathrm{GHz}$ and $v_{z} / v_{s}=100$, then $q_{z} v_{z} \tau=1$ for $\tau=10$ ps. As a comparison, the transport scattering time can be as long as $0.2 \mathrm{~ns}$ in the Dirac semimetal $\mathrm{Cd}_{3} \mathrm{As}_{2}$ [51]. Therefore, it is necessary to consider both $q_{z} v_{z} \tau \ll 1$ and $q_{z} v_{z} \tau \gg 1$ limits.

The intraband contribution to the magnetization in the $\omega \tau \ll 1$ and $q_{z} v_{z} \tau \ll 1$ limit is

$$
M_{z}^{\text {intra }} \approx M_{0}\left[1-\frac{3}{5}\left(q_{z} v_{z} \tau\right)^{2}\right] \mu \omega \tau^{2}
$$

and in the $\omega \tau \ll 1$ and $q_{z} v_{z} \tau \gg 1$ limit, we derive

$$
M_{z}^{\text {intra }} \approx 3 M_{0} \frac{1}{\left(q_{z} v_{z} \tau\right)^{2}}\left[1-\frac{\pi}{2\left(q_{z} v_{z} \tau\right)}\right] \mu \omega \tau^{2}
$$

Here, $M_{0}=e v_{x} v_{y}\left(\beta b u_{0} q_{z}\right)^{2} /\left(48 \pi^{2} v_{z}\right) \propto\left(u_{0} \omega\right)^{2} \propto I$, where $I$ is the intensity of the sound (see, e.g., Ref. [52]). In Eq. (9), we have expanded the result up to $\omega\left(q_{z} v_{z}\right)^{2}$ and omitted the subleading term $\propto \omega^{3}$. To obtain the above results, we assumed that $\mu \tau$ is large enough and $q_{z} v_{z} \tau \ll \mu \tau$. (See the SM [42] for details.) The interband contribution becomes important only for a small chemical potential or a short relaxation time $\mu \tau \ll 1$.

At large frequencies $\left(q_{z} v_{z} \tau \gg 1\right)$, magnetization $M_{z}^{\text {intra }}$ scales as $\mu / \omega$, while for the small ones $\left(q_{z} v_{z} \tau \ll 1\right)$, $M_{z}^{\text {intra }} \propto \mu \omega \tau^{2}$. This scaling is different from the case of the IFE [12] and is related to a different frequency dependence of the axial gauge fields. It is worth emphasizing that the AMEE is different also in the strong dependence on the wave vector $q_{z}$, which is negligible for the IFE.

Another notable feature is related to the presence of a peak in the magnetization. The peak appears due to the momentum dependence of the magnetization. From Eqs. (9) and (10), we can see that the magnetization reaches a maximum at $\omega \tau \propto v_{s} / v_{z}$ and the peak value is proportional to $\mu \tau$. The scaling behavior and the appearance of a peak of the sound-induced magnetization at small frequencies are in drastic contrast to the IFE, where the magnetization is proportional to $1 / \omega^{3}$ in the clean limit and to $\tau^{2} / \omega$ in the dirty limit. The scaling of the AMEE with $\omega$ should be robust as long as the strains can be interpreted as effective axial gauge fields and the deviations from the relativisticlike spectrum are small.

To estimate the effect, we use the following parameters for the Dirac semimetal $\mathrm{Cd}_{3} \mathrm{As}_{2}$ [53-55]: $v_{x}=8.47 \mathrm{eV} \AA, v_{y}=8.56 \mathrm{eV} \AA, v_{z}=2.16 \mathrm{eV} \AA, b=$ $0.16 \AA^{-1}$, and $v_{s}=1.6 \times 10^{3} \mathrm{~m} / \mathrm{s}$, which is about 200 times smaller than $v_{z}$. The chemical potential is fixed to be $0.2 \mathrm{eV}$. We further assume that $\beta=1$ [56] and the sound intensity is $I=10 \mathrm{~W} / \mathrm{cm}^{2}$ (see, e.g., Refs. [58,59]). In Fig. 4, we show the numerical results for the frequency dependence of the magnetization at different scattering

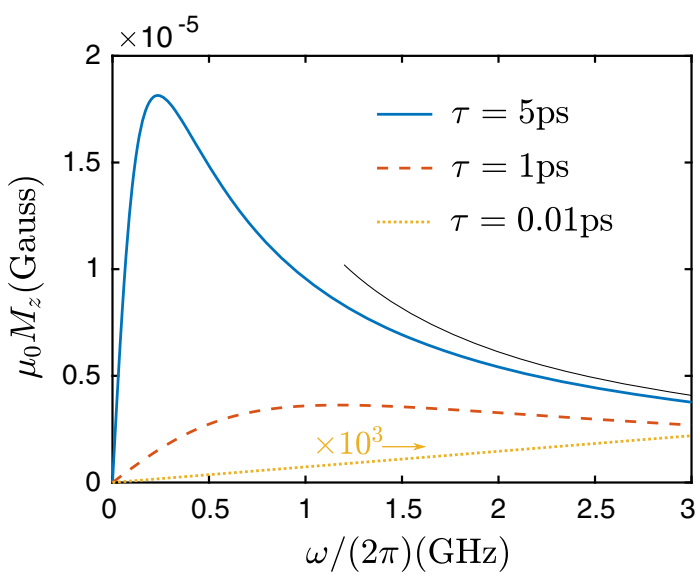

FIG. 4. The frequency dependence of the induced magnetization for $\mathrm{Cd}_{3} \mathrm{As}_{2}$ for different scattering times at the fixed sound intensity. Here, $\mu_{0}$ is the vacuum permeability. The results for $\tau=0.01 \mathrm{ps}$ are multiplied by $10^{3}$. The chemical potential is taken to be $0.2 \mathrm{eV}$, and the sound intensity is $10 \mathrm{~W} / \mathrm{cm}^{2}$. The black thin curve shows the magnetization calculated using the first term in Eq. (10).

times. The black thin line is plotted using the first term in Eq. (10): $M_{z}=3 M_{0} \mu \omega /\left(q_{z} v_{z}\right)^{2} \propto 1 / \omega$.

For $\tau=0.01 \mathrm{ps}$, which is of the same order as the quantum lifetime measured in $\mathrm{Cd}_{3} \mathrm{As}_{2}$ [51], the magnetization scales linearly with $\omega$ and the slope is proportional to $\tau^{2}$, and for $\tau=5 \mathrm{ps}$, it scales as $1 / \omega$ for $\omega /(2 \pi) \gtrsim 2 \mathrm{GHz}$. The peak is clearly observed for $\tau=5$ ps. The positions and heights of the peaks are in agreement with our estimates in Eqs. (9) and (10). For $\tau=1 \mathrm{ps}$ at $\omega /(2 \pi)=0.2 \mathrm{GHz}$, the generated magnetic field strength is $\mu_{0} M_{z} \approx 1 \mu \mathrm{G}$. Thus, the magnetic flux through a sample of diameter $1 \mathrm{~mm}$ is about one-tenth of the flux quantum, which is 5 orders above the SQUID threshold [60,61]. With the decrease of $\tau$, the magnetization decreases significantly, but it is still detectable even for $\tau=0.01$ ps at $\omega /(2 \pi)=$ $0.1 \mathrm{GHz}$. The interband correction to the magnetization for $\tau=1 \mathrm{ps}$ and $\tau=5 \mathrm{ps}$ is negligible, while for $\tau=0.01 \mathrm{ps}$ it contributes about $7 \%$ of the total magnetization in the plotted frequency region. Therefore, as one might naively expect, for large chemical potentials, the interband effects play a minor role in the sound-induced processes.

Unlike the IFE, where the wave vector of light is negligibly small, $q_{z}$ can be of the same order as the Fermi wave vector $\mu / v_{z}$ for the AMEE. Thus, electrons can backscatter on the Fermi surface at $q_{z} v_{z} \sim 2 \mu$ for strong (pseudo)spin-flipping scattering potentials, giving rise to a second peak in the induced magnetization. (See the SM [42] for details.)

Conclusion.-We propose a dynamical axial magnetoelectric effect where a static magnetization is generated as a result of the transfer of the angular momentum of the axial gauge field $\mathbf{E}_{5}(t)$ to the orbital magnetization of the 
electron quasiparticles. The resulting magnetization is of the second order in dynamical fields and reads as $\mathbf{M} \sim \mathbf{E}_{5}(\omega) \times \mathbf{E}_{5}^{*}(\omega)$.

The proposed axial magnetoelectric effect is universal with respect to the origin of the dynamic axial fields and could appear in many systems. As an example, we propose to use dynamical deformations (sound) in Dirac and Weyl semimetals to excite these fields and generate static magnetization. We found that, unlike the IFE, the induced magnetization scales as $\omega(1 / \omega)$ rather than $1 / \omega\left(1 / \omega^{3}\right)$ for small (large) frequencies in the dirty limit and at fixed sound intensity.

By using realistic model parameters, we estimated the induced magnetization in the Dirac semimetal $\mathrm{Cd}_{3} \mathrm{As}_{2}$. Being within experimental reach, the effect provides a way to investigate unusual axial electromagnetic fields via conventional magnetometry techniques.

We are grateful to M. Geilhufe for useful discussions. This work was supported by Nordita, the University of Connecticut, and the European Research Council under the European Unions Seventh Framework ERC-2018-SYG 810451 HERO, VILLUM FONDEN via the Centre of Excellence for Dirac Materials (Grant No. 11744), and Knut and Alice Wallenberg foundation (Grant No. KAW 2019-0068 Dynamic Quantum Matter). P. O. S. acknowledges the support through the Yale Prize Postdoctoral Fellowship in Condensed Matter Theory.

[1] A. M. Turner and A. Vishwanath, Beyond band insulators: Topology of semi-metals and interacting phases, in Topological Insulators, edited by M. Franz and L. Molenkamp (Elsevier Science, Amsterdam, 2013), Chap. 11, pp. 293322, https://doi.org/10.1016/B978-0-444-63314-9.00011-1.

[2] O. Vafek and A. Vishwanath, Dirac fermions in solids: From high- $T_{c}$ cuprates and graphene to topological insulators and Weyl semimetals, Annu. Rev. Condens. Matter Phys. 5, 83 (2014).

[3] T. Wehling, A. Black-Schaffer, and A. Balatsky, Dirac materials, Adv. Phys. 63, 1 (2014).

[4] N. P. Armitage, E. J. Mele, and A. Vishwanath, Weyl and Dirac semimetals in three-dimensional solids, Rev. Mod. Phys. 90, 015001 (2018).

[5] L. P. Pitaevskii, Electric forces in a transparent dispersive medium, J. Exp. Theor. Phys. 12, 1008 (1960), http://www .jetp.ac.ru/cgi-bin/ dn/e_012_05_1008.pdf.

[6] P. S. Pershan, Nonlinear optical properties of solids: Energy considerations, Phys. Rev. 130, 919 (1963).

[7] J. P. Van Der Ziel, P. S. Pershan, and L. D. Malmstrom, Optically-Induced Magnetization Resulting from the Inverse Faraday Effect, Phys. Rev. Lett. 15, 190 (1965).

[8] P. S. Pershan, J. P. Van Der Ziel, and L. D. Malmstrom, Theoretical discussion of the inverse Faraday effect, Raman scattering, and related phenomena, Phys. Rev. 143, 574 (1966).
[9] M. Battiato, G. Barbalinardo, and P. M. Oppeneer, Quantum theory of the inverse Faraday effect, Phys. Rev. B 89, 014413 (2014).

[10] K. Taguchi, T. Imaeda, M. Sato, and Y. Tanaka, Photovoltaic chiral magnetic effect in Weyl semimetals, Phys. Rev. B 93, 201202(R) (2016).

[11] A. A. Zyuzin, M. Silaev, and V. A. Zyuzin, Nonlinear chiral transport in Dirac semimetals, Phys. Rev. B 98, 205149 (2018).

[12] I. D. Tokman, Q. Chen, I. A. Shereshevsky, V. I. Pozdnyakova, I. Oladyshkin, M. Tokman, and A. Belyanin, Inverse Faraday effect in graphene and Weyl semimetals, Phys. Rev. B 101, 174429 (2020).

[13] M. Kawaguchi, H. Hirose, Z. Chi, Y.-C. Lau, F. Freimuth, and M. Hayashi, Giant inverse Faraday effect in Dirac semimetals, arXiv:2009.01388.

[14] Y. Gao, C. Wang, and D. Xiao, Topological inverse Faraday effect in Weyl semimetals, arXiv:2009.13392.

[15] Y. Aharonov and D. Bohm, Significance of electromagnetic potentials in the quantum theory, Phys. Rev. 115, 485 (1959).

[16] Y. Aharonov and A. Casher, Topological Quantum Effects for Neutral Particles, Phys. Rev. Lett. 53, 319 (1984).

[17] H. Suzuura and T. Ando, Phonons and electron-phonon scattering in carbon nanotubes, Phys. Rev. B 65, 235412 (2002).

[18] M. A. H. Vozmediano, M. I. Katsnelson, and F. Guinea, Gauge fields in graphene, Phys. Rep. 496, 109 (2010).

[19] J.-H. Zhou, H. Jiang, Q. Niu, and J.-R. Shi, Topological invariants of metals and the related physical effects, Chin. Phys. Lett. 30, 027101 (2013).

[20] A. Cortijo, Y. Ferreirós, K. Landsteiner, and M. A. H. Vozmediano, Elastic Gauge Fields in Weyl Semimetals, Phys. Rev. Lett. 115, 177202 (2015).

[21] D. I. Pikulin, A. Chen, and M. Franz, Chiral Anomaly from Strain-induced Gauge Fields in Dirac and Weyl Semimetals, Phys. Rev. X 6, 041021 (2016).

[22] A. G. Grushin, J. W. F. Venderbos, A. Vishwanath, and R. Ilan, Inhomogeneous Weyl and Dirac Semimetals: Transport in Axial Magnetic Fields and Fermi Arc Surface States from Pseudo-Landau Levels, Phys. Rev. X 6, 041046 (2016).

[23] R. Ilan, A. G. Grushin, and D. I. Pikulin, Pseudoelectromagnetic fields in 3D topological semimetals, Nat. Rev. Phys. 2, 29 (2020).

[24] C.-X. Liu, P. Ye, and X.-L. Qi, Chiral gauge field and axial anomaly in a Weyl semimetal, Phys. Rev. B 87, 235306 (2013).

[25] Y. Araki, Magnetic textures and dynamics in magnetic Weyl semimetals, Ann. Phys. (Amsterdam) 532, 1900287 (2020).

[26] N. Levy, S. A. Burke, K. L. Meaker, M. Panlasigui, A. Zettl, F. Guinea, A. H. Castro Neto, and M. F. Crommie, Straininduced pseudo-magnetic fields greater than 300 tesla in graphene nanobubbles, Science 329, 544 (2010).

[27] Y. Liu, J. N. B. Rodrigues, Y. Z. Luo, L. Li, A. Carvalho, M. Yang, E. Laksono, J. Lu, Y. Bao, H. Xu, S. J. R. Tan, Z. Qiu, C. H. Sow, Y. P. Feng, A. H. C. Neto, S. Adam, J. Lu, and K. P. Loh, Tailoring sample-wide pseudo-magnetic fields on a grapheneblack phosphorus heterostructure, Nat. Nanotechnol. 13, 828 (2018). 
[28] P. Nigge, A. C. Qu, Lantagne-Hurtubise, E. Mårsell, S. Link, G. Tom, M. Zonno, M. Michiardi, M. Schneider, S. Zhdanovich, G. Levy, U. Starke, C. Gutiérrez, D. Bonn, S. A. Burke, M. Franz, and A. Damascelli, Room temperature strain-induced Landau levels in graphene on a waferscale platform, Sci. Adv. 5, eaaw5593 (2019).

[29] C. C. Hsu, M. L. Teague, J. Q. Wang, and N. C. Yeh, Nanoscale strain engineering of giant pseudo-magnetic fields, valley polarization, and topological channels in graphene, Sci. Adv. 6, eaat9488 (2020).

[30] B. Z. Spivak and A. V. Andreev, Magnetotransport phenomena related to the chiral anomaly in Weyl semimetals, Phys. Rev. B 93, 085107 (2016).

[31] P. Rinkel, P. L. S. Lopes, and I. Garate, Influence of Landau levels on the phonon dispersion of Weyl semimetals, Phys. Rev. B 99, 144301 (2019).

[32] P. O. Sukhachov and H. Rostami, Acoustogalvanic Effect in Dirac and Weyl Semimetals, Phys. Rev. Lett. 124, 126602 (2020).

[33] L.-L. Gao, S. Kaushik, D. E. Kharzeev, and E. J. Philip, Chiral kinetic theory of anomalous transport induced by torsion, arXiv:2010.07123.

[34] V.E. Lyamov, Polarization Effects and Anisotropy of Acoustic Wave Interaction in Crystals [in Russian] (MGU, Moscow, 1983).

[35] B. A. Auld, C. F. Quate, H. J. Shaw, and D. K. Winslow, Acoustic quarter-wave plates at microwave frequencies, Appl. Phys. Lett. 9, 436 (1966).

[36] H. van de Vaart and H. I. Smith, High efficiency polarization reversal of magnetoelastic waves in YIG by optical-contact bonding of YAG disks, Appl. Phys. Lett. 9, 439 (1966).

[37] V. V. Lemanov, A. V. Pavlenko, and A. N. Grishmanovskii, Interaction between elastic and spin waves in yttrium iron garnet crystals, Sov. Phys. JETP 32, 389 (1971), http://jetp .ac.ru/cgi-bin/ dn/e_032_03_0389.pdf.

[38] D. M. Juraschek and N. A. Spaldin, Orbital magnetic moments of phonons, Phys. Rev. Mater. 3, 064405 (2019).

[39] In the case where circularly polarized phonons are induced optically, a conventional IFE should also be taken into account. Since the decay depths and rates of photons and phonons are different, the AMEE and the IFE can be distinguished in nonlocal and time-dependent probes.

[40] B. Cheng, T. Schumann, Y. Wang, X. Zhang, D. Barbalas, S. Stemmer, and N. P. Armitage, A large effective phonon magnetic moment in a dirac semimetal, Nano Lett. 20, 5991 (2020).

[41] E. J. Sie et al., An ultrafast symmetry switch in a Weyl semimetal, Nature (London) 565, 61 (2019).

[42] See Supplemental Material, which contains Refs. [43-49], at http://link.aps.org/supplemental/10.1103/PhysRevLett .126.247202 for details of derivations of the axial magnetoelectric effect and the results for Weyl and two-dimensional Dirac semimetals.

[43] J. Shi, G. Vignale, D. Xiao, and Q. Niu, Quantum Theory of Orbital Magnetization and Its Generalization to Interacting Systems, Phys. Rev. Lett. 99, 197202 (2007).
[44] L. Liang, T. I. Vanhala, S. Peotta, T. Siro, A. Harju, and P. Törmä, Band geometry, Berry curvature, and superfluid weight, Phys. Rev. B 95, 024515 (2017).

[45] E. V. Gorbar, V. A. Miransky, I. A. Shovkovy, and P. O. Sukhachov, Origin of Bardeen-Zumino current in lattice models of Weyl semimetals, Phys. Rev. B 96, 085130 (2017).

[46] A. Altland and B. D. Simons, Condensed Matter Field Theory, 2nd ed. (Cambridge University Press, Cambridge, England, 2010).

[47] Z. Wang, H. Weng, Q. Wu, X. Dai, and Z. Fang, Threedimensional Dirac semimetal and quantum transport in $\mathrm{Cd}_{3} \mathrm{As}_{2}$, Phys. Rev. B 88, 125427 (2013).

[48] M. V. Berry, Quantal phase factors accompanying adiabatic changes, Proc. R. Soc. A 392, 45 (1984).

[49] J. P. Provost and G. Vallee, Riemannian structure on manifolds of quantum states, Commun. Math. Phys. 76, 289 (1980).

[50] M. E. Peskin and D. V. Schroeder, An Introduction to Quantum Field Theory (Addison-Wesley, Reading, 1995).

[51] T. Liang, Q. Gibson, M. N. Ali, M. Liu, R. J. Cava, and N. P. Ong, Ultrahigh mobility and giant magnetoresistance in the Dirac semimetal $\mathrm{Cd}_{3} \mathrm{As}_{2}$, Nat. Mater. 14, 280 (2015).

[52] A. A. Abrikosov, Fundamentals of the Theory of Metals (North-Holland, Amsterdam, 1988).

[53] Z. K. Liu, J. Jiang, B. Zhou, Z. J. Wang, Y. Zhang, H. M. Weng, D. Prabhakaran, S.-K. Mo, H. Peng, P. Dudin, T. Kim, M. Hoesch, Z. Fang, X. Dai, Z. X. Shen, D. L. Feng, Z. Hussain, and Y.L. Chen, A stable three-dimensional topological Dirac semimetal $\mathrm{Cd}_{3} \mathrm{As}_{2}$, Nat. Mater. 13, 677 (2014).

[54] H. Wang, Y. Xu, M. Shimono, Y. Tanaka, and M. Yamazaki, Computation of interfacial thermal resistance by phonon diffuse mismatch model, Mater. Trans., JIM 48, 2349 (2007).

[55] Non-Tetrahedrally Bonded Elements and Binary Compounds I, edited by O. Madelung, U. Rossler, and M. Schulz (Springer-Verlag, Berlin Heidelberg, 1998).

[56] Grüneisen constants are relatively material independent and are of order 1-2 [57].

[57] H. Ibach and H. Lüth, Solid-State Physics: An Introduction to Theory and Experiment (Springer Berlin Heidelberg, Berlin Heidelberg, 2012).

[58] G. Weinreich and H. G. White, Observation of the acoustoelectric effect, Phys. Rev. 106, 1104 (1957).

[59] P. Smith and D. O. Sproule, Experiments on the acoustoelectric effect, Nature (London) 184, 264 (1959).

[60] M. Buchner, K. Höfler, B. Henne, V. Ney, and A. Ney, Tutorial: Basic principles, limits of detection, and pitfalls of highly sensitive SQUID magnetometry for nanomagnetism and spintronics, J. Appl. Phys. 124, 161101 (2018).

[61] C. Granata and A. Vettoliere, Nano superconducting quantum interference device: A powerful tool for nanoscale investigations, Phys. Rep. 614, 1 (2016). 